\title{
«Ein Spielen der Liebe mit sich selbst». Hegel e la teoria spinoziana dell'amor Dei intellectualis
}

\section{«Ein Spielen der Liebe mit sich selbst». Hegel and Spinoza's doctrine of amor dei intellectualis}

RESUMEN: Nella parte quinta dell'Etica, Spinoza descrive l'«amore intellettuale verso Dio» - che nasce nella mente che ha raggiunto la scientia intuitiva - come una "parte dell'amore infinito con il quale Dio ama se stesso». Scopo del saggio è gettare luce sui rilevanti, ma scarsamente considerati, riferimenti di Hegel alla teoria dell'amor dei intellectualis, tentando di ricostruirne il significato a due livelli differenti: anzitutto, esaminando le Lezioni sulla storia della filosofia, dove la descrizione dell'amore intellettuale è inserito in una disamina storico-filosofica del pensiero spinoziano; in secondo luogo, analizzando alcuni passaggi della Fenomenologia dello spirito e della Scienza della logica, opere in cui Hegel richiama l'amor dei intellectualis per esprimere metaforicamente la convinzione che l'Assoluto va concepito in termini dialettici, come soggetto e spirito.

PALABRAS CLAVE: HEGEL - SPINOZA AMOR DEI INTELLECTUALIS

\begin{abstract}
In the fifth part of the Ethica Spinoza describes the «intellectual love toward God» - arising in the mind that has achieved the scientia intuitiva - as a "part of the infinite love wherewith God loves himself». The aim of the essay is to cast light, at two different levels, on the interesting but rarely studied Hegel's references to the theory of amor dei intellectualis: firstly, focusing on the Lectures on the History of Philosophy, where the description of intellectual love is included in an historico-philosophical account of Spinoza's thought; secondly, dealing with the Phenomenology of Spirit and in the Science of Logic, works in which Hegel mentions the amor dei intellectualis in order to express the idea that the Absolute has to be conceived in dialectical terms, as subject and spirit.

KEY WORDS: HEGEL - SPINOZA AMOR DEI INTELLECTUALIS
\end{abstract}




\section{Hegel tra SPINOZISMO ED INTERPRETAZIONE Di SPINOZA}

I .1 Nella Prefazione al suo volume del 1988 intitolato Platonisme et interprétation de Platon a l'époque moderne, J.-L. Vieillard-Baron sottolineava la necessità, nell'avvicinarsi allo studio della fortuna di Platone, di distinguere tra "platonisme», intesa come l'«assimilation» della filosofia platonica «à l'intérieur d'un pensée autre» e l'«interprétation de Platon proprement dite», il cui scopo è restituire in qualche modo un' immagine del pensiero platonico legata direttamente al testo dei dialoghi. ${ }^{1}$ Questa distinzione di piani era ritenuta particolarmente valida anche a proposito di Hegel; al tempo stesso, però, date le premesse e le peculiarità metodologiche delle Lezioni sulla storia della filosofia, ${ }^{2}$ il livello dell'assimilazione dei concetti elaborati da altri filosofi e quello della loro interpretazione storico-filosofica si rivelano in realtà indisgiungibili ed Hegel, nell'esporre i sistemi e le posizioni teoriche dei pensatori che l'hanno preceduto, finisce spesso col valorizzare autori ed idee che maggiore influenza hanno avuto nella nascita e nell'evoluzione della propria filosofia. Queste considerazioni si rivelano molto efficaci anche se applicate al rapporto che Hegel instaura con Spinoza, figura che, fin dalla lettura delle Lettere sulla dottrina di Spinoza di Jacobi risalente agli anni trascorsi presso lo Stift di Tubinga, ${ }^{3}$

[1] J.L. Vieillard-Baron, Platonisme et interprétation de Platon a lépoque moderne, Paris, Vrin, 1988, p. 7

[2] Scrive Hegel introducendo le proprie Lezioni che la tradizione filosofica non rappresenta solo «una massaia, che custodisce fedelmente ciò che ha ricevuto per conservarlo e trasmetterlo invariato ai posteri, come fa il corso della natura, che nelle infinite variazioni e attività delle sue manifestazioni e funzioni, rimane sempre ferma nelle sue leggi originali e non compie alcun progresso». Diversamente, essa «è viva e s'ingrandisce come un fiume impetuoso che cresce man mano, quanto più si allontana dalla sua sorgente». In questo fiume impetuoso, ciascuna filosofia rappresenta l'inveramento di quella che l'ha preceduta, in una successione di sistemi ciascuno dei quali nega ed al tempo stesso contiene quelli a sé precedenti: «Questa è appunto la posizione e la funzione della nostra età, come quella d’ogni età: afferrare la scienza già esistente, fermarsi su di essa e in tal modo svolgerla e innalzarla a un grado superiore. Mentre ce ne appropriamo, le aggiungiamo qualcosa di nostro, che prima non aveva. Da questa struttura della produzione spirituale, che presuppone un mondo spirituale preesistente e lo trasforma appropriandosene, deriva come conseguenza che può sorgere una nostra filosofia, solo in quanto si ricollega a quella precedente, di cui è il necessario risultato. Perciò il corso della storia non ci mostra il divenire di cose a noi estranee, bensì il nostro stesso divenire, il divenire della nostra scienza» (G.W.F. Hegel, Introduzione alla storia della filosofia, tr. A. Plebe, Bari, Laterza, 1962, pp. 54-55). La filosofia hegeliana, nella fattispecie, occupa una posizione peculiare nella misura in cui incarna la forma più alta, e presumibilmente definitiva, del sapere filosofico, identificandosi, de facto, con lo stesso sapere assoluto nel suo concretizzarsi storico.

[3] cfr. F. Chiereghin, L'influenza dello spinozismo nella formazione della filosofia hegeliana, 
occupa un posto speciale sullo scrittoio del filosofo svevo; testimonianza assai significativa dell'importanza di Spinoza nella genesi dell'idealismo hegeliano è in questo senso proprio l'ampia sezione delle Lezioni sulla storia della filosofia dedicata al pensatore olandese, nel corso della quale Hegel afferma:

[...], la sostanza assoluta è il vero, ma non l'intero vero; per esser tale, essa dovrebbe pensarsi anche come attiva e vivente in se stessa, e così appunto determinarsi come spirito. Invece la sostanza spinoziana è soltanto la determinazione generale, e quindi astratta, dello spirito. Si può dire, è vero, che questo pensiero è la base di ogni vera veduta, ma non come fondamento che stia assolutamente fermo di sotto, sibbene come quella unità astratta, che lo spirito è dentro di sé. Va dunque rilevato che il pensiero non poteva non porsi dal punto di vista dello spinozismo: essere spinoziani è l'inizio essenziale del filosofare. Infatti, [...], non si comincia a filosofare, senza che l'anima si tuffi anzitutto in quest'etere dell'unica sostanza, in cui è sommerso tutto quel che si era ritenuto vero; questa negazione di tutto quel ch'è particolare cui deve esser pervenuto ogni filosofo è la liberazione dello spirito e la sua base assoluta. ${ }^{4}$

Nella misura in cui consente di accedere al punto di vista della «sostanza assoluta», di quell'«unità astratta» che è al tempo stesso negazione e fondamento «di tutto quel ch'è particolare», il pensiero di Spinoza incarna «l'inizio essenziale del filosofare», il presupposto inderogabile da cui deve prendere le mosse «ogni filosofo». Al tempo stesso, però, Hegel sottolinea la necessità di non arrestarsi a quella che è pur sempre una «determinazione generale, e quindi astratta, dello spirito»; nel mettere a fuoco l'unità della sostanza assoluta, infatti, il filosofo olandese ha intuito il vero, ma non l'«intero vero», non è giunto cioè all'individuazione della natura eminentemente spirituale e dialettica dell'unità del tutto. La sostanza di Spinoza non è infatti tale da «pensarsi anche come attiva e vivente in se stessa», non si determina "come spirito»; essa infatti annulla il finito ed il negativo, immergendolo «in quest'etere dell'unica sostanza», piuttosto che accoglierlo dentro di sé come momento necessario del proprio sviluppo. Rivelatorio, a tal proposito, è il modo in cui Spinoza ha inteso la negazione, concependola come soppressione unilaterale del particolare, annullato in un'unità indifferenziata che rimane astratta e non si traduce nel «principio della soggettività, dell'individualità, della personalità», non si arricchisce del «momento dell'autocoscienza nell'essenza». ${ }^{5}$ Pur avendo il merito di intuire

Padova, CEDAM, 1961, pp. 26-29; A. TAssi, Hegel a Berna: le premesse di un sistema, Soveria Mannelli, Rubbettino, 2003, pp. 62-63.

[4] G.W.F. Hegel, Lezioni sulla storia della filosofia, tr. E. Codignola e G. Sanna, 3 voll. in 4 tomi, Firenze, La Nuova Italia, 1981-1985, t. III.2, pp. 109-110.

[5] Ibid., p. 140. 
che il determinato, il particolare è una negazione dell'universale e pertanto può essere compreso solo nel suo riferirsi all'unità del tutto, un'intuizione compendiata nella formula omnis determinatio est negatio, ${ }^{6}$ Spinoza stesso non è stato poi in grado di esplorarne fino in fondo l'autentico significativo speculativo. Nella Scienza della logica, proponendosi quale continuatore del filosofo olandese, Hegel intende esporre l'inveramento di tale principio, ponendolo in relazione al movimento di negazione e particolarizzazione che scandisce la vita dell'assoluto: la negazione che collocava il finito al di fuori dell'assoluto, relegandolo nella sfera del semplice non-essere, viene completata con una seconda negazione che la tramuta nel processo di riflessione mediante il quale il finito, il determinato non è più annullato, ma negato in modo tale da essere conservato nell'universale quale momento necessario del suo sviluppo. ${ }^{7}$ La convinzione, espressa nelle Lezioni, che la speculazione spinoziana identifichi il luogo germinale di qualsiasi autentica speculazione filosofica, trova così conferma nello stesso modus operandi adottato da Hegel, che sempre nella Scienza della logica non manca di sottolineare che il «punto di vista della sostanza» descritto nel «sistema di Spinoza» non può essere semplicemente eluso o confutato, ma va riconosciuto «anzitutto come essenziale e necessario». ${ }^{8}$

I.2 Nelle pagine che seguono si cercherà di mostrare come Hegel ricorra, proprio per esporre la concezione spirituale e dialettica dell'Assoluto che della sostanza spinoziana si propone quale superamento, ad un'altra dottrina elaborata dal filosofo olandese, quella dell'amor dei intellectualis. In più di un'occasione, infatti, Hegel fa riferimento alle celebri battute della parte quinta dell'Etica, in cui Spinoza descrive l'amore dell'uomo verso Dio, scaturente dalla visione dell'ordine necessario di tutte le cose consentita dal terzo e supremo genere di conoscenza, quella intuitiva, che schiude all'individuo la comprensione della propria appartenenza all'unica «realtà immutabile ed eterna». ${ }^{9}$ Accompagnato da una gioia eterna, l'amore dell'uomo per Dio rappresenta la realizzazione, a livello individuale, dell'unico amore che Dio prova per se stesso nella misura in

[6] Ibid., pp. 120-121, p. 140: «Spinoza ha enunciato la grande proposizione che ogni determinazione racchiude in se stessa negazione». L'espressione è contenuta nella lettera di Spinoza a Jelles del 2 giugno 1674, cfr. B. SpINOzA, Epistolario, tr. A. Droetto, Torino, Giulio Einaudi editore, 1951, pp. 225-226.

[7] G.W.F. Hegel, Scienza della logica, tr. A. Moni, 2 voll., Laterza, Bari, 2004, vol. 1, pp. 108-109. Si veda, a questo proposito, l'illuminante Y.Y. Melamed, "Omnis determinatio est negatio": Determination, Negation and Self-Negation in Spinoza, Kant, and Hegel», in E. Förster e Y.Y. Melamed, Spinoza and German Idealism, Cambridge, Cambridge University Press, 2002, pp. 175-196.

[8] Hegel, Scienza della logica, vol. 2, p. 655.

[9] B. SpInoza, Etica, in Id., Opere, Milano, Mondadori, 2007, parte V, prop. XX, scol., p. 1069. 
cui è in grado di intendersi perfettamente quale causa del tutto. Gli individui, quando amano quel Dio col quale condividono un'unica essenza, diventano parte consapevole dell'unica attività con cui la sostanza divina incessantemente afferma se stessa:

L'amore intellettuale della mente verso Dio - scrive Spinoza - è lo stesso amore di Dio, con il quale Dio ama sé stesso, non in quanto è infinito, ma in quanto può essere esplicato mediante l'essenza della mente umana considerata sotto l'aspetto dell'eternità (sub specie aeternitatis); cioè, l'amore intellettuale della mente verso Dio è parte dell'amore infinito con il quale Dio ama se stesso. ${ }^{10}$

La ricostruzione storico-filosofica che Hegel opera della teoria esposta in queste battute sarà oggetto dalla prima parte di questo lavoro, incentrata soprattutto sulle Lezioni sulla storia della filosofia, dove l'amor dei intellectualis è ricondotto direttamente al monismo sostanzialistico esposto nella prima parte dell'Etica ed è analizzato come espressione di un modo di ragionare che costantemente spinge Spinoza a «ricondurre tutte le limitazioni alla sostanza anziché fissare il singolo»; ${ }^{11}$ nella fattispecie, la massima felicità e libertà dell'individuo vengono fatte risiedere nel suo volgersi a Dio, nel prendere coscienza della propria totale appartenenza ad un unico tutto. L'amor dei intellectualis è in tal senso il culmine di una morale perfettamente coerente con un'ontologia che non conferisce al particolare alcuna sussistenza autonoma rispetto all'universale. Ad un livello ulteriore, però, Hegel scorge nell'attribuzione a Dio di un amore verso se stesso, e nella sua identificazione con l'amore dell'uomo verso Dio, un tentativo di articolare e vivificare la staticità della sostanza; al pari del principio omnis determinatio est negatio, quindi, la dottrina dell'amor dei intellectualis rappresenterebbe un'intuizione con cui Spinoza si pone, per così dire, al di là dei limiti della propria filosofia e che, estrapolata dal proprio contesto originario, può dispiegare il proprio potenziale speculativo. Ė quanto accade nella Fenomenologia dello spirito e nella Scienza della logica, dove Hegel evoca la parte quinta dell'Etica per esprimere una concezione dell'Assoluto che proprio del sostanzialismo spinoziano, nonché della metafisica di Schelling che ad esso viene associata, intende essere il cosciente superamento. Sarà questa riappropriazione l'oggetto della seconda parte del lavoro, al termine del quale l'interpretazione storico-filosofica del pensiero di Spinoza e l'assunzione critica che Hegel ne opera si paleseranno come due poli tra i quali agisce una feconda tensione teorica; verrà in questo modo chiarito uno dei risvolti più affascinanti

[10] Ibid., prop. XXXVI, p. 1079.

[11] HegeL, Lezioni sulla storia della filosofia, t. III.2, p. 129. 
ma meno studiati dell'articolato dialogo che il pensatore svevo intrattiene, nell'intero arco della sua riflessione filosofica, con l'autore dell'Etica. ${ }^{12}$

\section{SPINOZA E LA «MORALE PIÙ SUBLIME»}

Nelle Lezioni sulla storia della filosofia l'amore intellettuale di Dio è presentato quale coronamento della «morale più sublime» che Spinoza ha innestato sul proprio rigoroso monismo, nel cui ambito la massima libertà dell'uomo risiede nel possesso della vera idea di Dio. Lo spirito finito è pertanto morale nella misura in cui «indirizza a Dio la sua conoscenza e la sua volontà, poiché la verità è la conoscenza di Dio». ${ }^{13}$ Alla vera idea di Dio si perviene tramite il terzo grado di conoscenza, quella scientia intuitiva che, riassume Hegel, fa seguito, dapprima, all'«opinione e immaginazione», in cui «rientra tutto quello che noi conosciamo delle cose singole mediante i sensi, in maniera mutila e disordinata, e poi da indizi, rappresentazioni, ricordi», in secondo luogo, a quella conoscenza che «attingiamo da concetti universali e da idee adeguate delle proprietà delle cose». Grazie alla scienza intuitiva, invece, le cose vengono ricondotte al loro fondamento eterno e pensate «sotto una certa forma d'eternità»: «dall'idea adeguata di alcuni attributi di Dio» si giunge «alla conoscenza adeguata dell'essenza delle cose» e, al contempo, in ciascuna di queste viene riconosciuta la presenza dell'eterna e infinita essenza di Dio; ${ }^{14}$ «L'uomo deve far risalire tutto a Dio, poiché Dio è un uno in tutto; soltanto l'eterna essenza di Dio $\dot{e}$, soltanto l'eterna verità l'uomo persegue nel suo operare» ${ }^{15}$. Punto essenziale, raggiungendo il terzo genere di conoscenza l'uomo riconosce anche se stesso quale parte finita di un tutto infinito: «Questo è il ritorno dello spirito a Dio, ed è la libertà umana; invece in quanto modus lo spirito non ha libertà, ma è determinato da altro». ${ }^{16}$ Dalla conquista di questa libertà - che, risentendo dei

[12] Sulla questione si segnalano due contributi i cui risultati verranno assunti, nelle pagine che seguono, come un punto di partenza: W. JANKE, «Amor Dei intellectualis. Vernunft- und Gottesliebe in Gipfelsätzen neuzeitlicher Systembildungen (Spinoza, Hegel, Schelling Fichte)», Daimon. Revista de Filosofia, IX (1994), pp. 101-114; A. Nuzzo, «Spinozas Amor dei intellectualis und Hegels methodologische Umdeutung des Liebesbegriffs», in: Affekte ohne Norm. Spinozas Affektenlehre im historischen und aktuellen Kontext, ed. by A. ENGSTLER, R. SCHNEPF, Hildesheim, Olms Verlag, 2002, pp. 246-262: 259

[13] HegeL, Lezioni sulla storia della filosofia, t. III.2, p. 129.

[14] Il riferimento qui è alla prop. XLV della seconda parte dell'Etica, in cui Spinoza osserva che «ciascuna idea di qualunque cosa esistente in atto implica necessariamente l'essenza eterna e infinita di Dio» (B. Spinoza, Etica, p. 883), proposizione ripresa nello scolio alla prop. XXIX della parte quinta, dove si osserva che questo tipo di consapevolezza coincide con la capacità di concepire le cose «sotto l'aspetto dell'eternità» (ibid., p. 1075).

[15] Hegel, Lezioni sulla storia della filosofia, t. III.2, p. 131.

[16] Ibid. 
limiti propri della metafisica spinoziana, non è per Hegel la libertà autentica dello spirito che si determina da sé, ma si traduce nella consapevolezza della ferrea necessità cosmica cui l'esistenza di ciascun individuo è vincolata - nasce «l'amore intellettuale di Dio»; assumendo il punto di vista dell'eternità e della necessità, l'uomo sente sorgere in sé un "amore infinito, intellettuale» che si identifica con l'amore che Dio prova per se stesso nella misura in cui "può avere per suo fine e per sua causa soltanto se stesso». ${ }^{17}$ La morale spinoziana è per Hegel la "più pura» poiché conduce alla comprensione che tutto ciò che è sensibile e finito altro non è che una limitazione, che trova la propria ragion d'essere in una sostanza infinita la cui contemplazione schiude all'uomo quella «liberazione» dal particolare che è poi ciò che fa dello spinozismo l'inizio del filosofare. ${ }^{18}$ L'identificazione finale tra uomo e Dio che Hegel vede tracciata nelle pagine conclusive dell'Etica, identificazione che è appunto imperniata sulla riconduzione del finito all'infinito, non si accompagna però alla comprensione che il primo non viene semplicemente annullato nel secondo ed incarna un momento necessario di una vita che va intesa come processo e sviluppo; anche la dottrina dell'amor dei intellectualis, in questo senso, si rivela condizionata dall'unilateralità della metafisica di Spinoza, esplicitata da Hegel nelle battute conclusive della propria esposizione:

Poiché nell'unica sostanza tutte le differenze e determinazioni delle cose e della coscienza non fanno che retrocedere, si può dire che nel sistema spinoziano non si fa altro che gettar tutto in questo abisso dell'annientamento. Ma nulla ne esce; e il particolare, di cui parla Spinoza, è soltanto trovato, preso dalla rappresentazione, senza essere giustificato. Per giustificarlo, Spinoza dovrebbe dedurlo dalla sua sostanza; ma questa non si schiude, e non perviene quindi ad alcuna vita, spiritualità ed attività. La sua filosofia ha soltanto la rigida sostanza, non ancora lo spirito; in essa non si è presso di sé. ${ }^{19}$

Nell'intendere il particolare ed il determinato - e quindi anche «l'anima, lo spirito» dell'individuo che perviene alla scientia intuitiva - come mera negazione, Spinoza si è precluso la possibilità di conferire alla sostanza «vita, spiritualità ed attività»; è per questo che alla sua metafisica manca «il principio della soggettività, dell'individualità, della personalità, il momento dell'autocoscienza nell'essenza». Il rivolgersi dell'uomo a Dio rimane un momento esteriore rispetto alla vita di un Assoluto che è del tutto «distinto dalla natura e dalla coscienza umana». Eppure, l'attribuzione a Dio di un amore verso se stesso e l'unione con la divinità cui l'uomo approda grazie all'amor dei intellectualis

[17] Ibid., p. 132.

[18] Ibid., p. 110

[19] Ibid., p. 142 
sembrano tendere in una direzione che valica i limiti della stessa filosofia spinoziana, celando i primi indizi di un ripensamento in chiave spirituale e dialettica della sostanza. Inquadrando, a conclusione delle Lezioni, il pensiero di Spinoza nell'evoluzione della filosofia moderna, il cui compito è comprendere «come spirito» il «pensiero della totalità» messo in luce dai Neoplatonici, Hegel afferma: «Pensiero ed essere sono opposti ed identici in Spinoza, il quale ha l'intuizione sostanziale, ma il conoscere è esteriore alla sostanza». ${ }^{20}$ In questo senso, identificando l'amore dell'uomo verso Dio con quello di Dio verso se stesso, Spinoza offriva ad Hegel un efficace strumento proprio per mostrare che il conoscere è un processo strutturalmente connesso al farsi della sostanza, processo che ne rivela la natura di spirito e di soggetto. Se, come ribadito nelle Lezioni sulle prove dell'esistenza di Dio, l'idea che la «massima determinazione dell'uomo sia il suo volgersi a Dio, l'amore puro per la divinità che si trova nell'espressione di Spinoza sub specie aeterni» è del tutto conseguente con l'impianto metafisico dell'Etica, ${ }^{21}$ d'altro canto, la negazione unilaterale del particolare ha però impedito al filosofo olandese di articolare correttamente quel rapporto di unità dialettica tra finito ed infinito che proprio dall'amor dei intellectualis era suggerito e che lo stesso Hegel intende esporre, oltrepassando l'ambito della morale in direzione di un modo di rappresentare e concepire la vita dell'Assoluto che del sostanzialismo spinoziano intende superare i limiti.

\section{L'AMOR DEI INTELLECTUALIS \\ tra Fenomenologia e SCIENZA DELLA LOGICA}

Nell'assimilare la teoria dell'amor dei intellectualis Hegel intreccia i due aspetti intorno ai quali ruota la prospettiva delineata nella parte quinta dell' $E$ tica, ossia, da un lato, l'idea che in esso l'individuo si riconosca parte integrante dell'unica sostanza e, dall'altro, la convinzione che tale amore sia parte di quello infinito con cui Dio ama se stesso. Per Hegel, come accennato, si tratta di due nuclei concettuali che, se letti nella giusta chiave ed al di là dei limiti connessi alla posizione spinoziana, vanno direttamente in direzione di quella personalità e di quella soggettività estranee alla sostanza così come l'aveva teorizzata Spinoza. Ė una duplicità di piani ben presente nelle battute della Prefazione alla Fenomenologia che dà il titolo alla presente ricerca; polemizzando in particolare con la filosofia schellinghiana dell'identità, che proprio del sostanzialismo spinoziano pareva rappresentare un'infelice riedizione, Hegel espone la necessità di pensare l'Assoluto non come l'abisso del vuoto, in cui tutto è uguale, bensì di pervenire «alla conoscenza differenziante e compiuta, o che ricerca ed esige il

[20] Ibid., pp. 413-414.

[21] G.W.F. Hegel, Lezioni sulle prove dellesistenza di Dio, Brescia, Morcelliana, 2009, p. 240. 
proprio compimento»; il raggiungimento di tale risultato dipende innanzitutto dal «cogliere ed esprimere il vero non tanto come sostanza, bensì propriamente come soggetto». Concepire Dio come sostanza unica ed indifferenziata, osserva subito dopo Hegel alludendo direttamente a Spinoza, causò indignazione in quanti, non a torto, vi percepirono la possibilità che l'autocoscienza, lungi dal mantenersi, finiva solamente per soccombere. L'universalità semplice, la «sostanzialità immobile e indifferenziata» di Spinoza prima e di Schelling poi, ${ }^{22}$ rappresenta pertanto un punto di partenza sì necessario ma al quale non è possibile arrestarsi, pena l'incomprensione della natura 'vivente' della sostanza, la quale va compresa nella sua autentica natura di soggetto:

[...], la sostanza vivente costituisce l'essere che è veramente soggetto, che è veramente reale, solo nella misura in cui essa è il movimento del porre-se-stessa, solo in quanto è la mediazione tra il divenire-altro-da-sé e se stessa. In quanto soggetto, la sostanza è la negatività pura e semplice, e proprio per questo è lo sdoppiamento del semplice, è la duplicazione opponente che a sua volta costituisce la negazione di questa diversità indifferente e della sua opposizione: solo questa uguaglianza restaurantesi, solo questa riflessione entro se stesso nell'essere-altro - non un'unità originaria in quanto tale, né immediata in quanto tale - è il vero. Il vero è il divenire di se stesso, è il circolo che presuppone la propria fine come proprio fine, e che è reale solo mediante l'attuazione e la propria fine ${ }^{23}$.

Proprio per descrivere la natura di tale svolgimento, in cui l'Assoluto si estrania da sé per poi riconciliarsi con se stesso configurandosi perciò come un risultato, viene evocato l'amor dei intellectualis di Spinoza: «È dunque possibile esprimere la vita di Dio e la conoscenza divina come un gioco dell'amore con se stesso», un vita che non è «l'imperturbata uguaglianza e unità con se stessa», ma, come Hegel intende chiarire proprio evocando il legame dialettico tra unità e distinzione implicito nell'amore, va interpretata come uno svolgimento ed uno sviluppo che ha nel rapportarsi «con l'essere-altro e con l'estraniazione» così come «con il superamento di questa estraniazione» la propria cifra carattestica. Hegel assume perciò l'idea spinoziana dell'infinito amore di Dio verso se stesso, e dell'amore dell'uomo verso Dio che ne rappresenta una specificazione finita, per esprimere «il movimento del riflettersi verso se stesso» di un Tutto che «si compie mediante il proprio sviluppo. Dell'Assoluto, infatti, bisogna dire che è essenzialmente un risultato, che solo alla fine è ciò che è in verità. E appunto in ciò consiste la sua natura: nell'essere realtà, soggetto, divenire-se-stesso» ${ }^{24}$. L'identificazione tra uomo e Dio descritta nella quinta parte dell'Etica viene

[22] G.W.F. Hegel, Fenomenologia dello Spirito, tr. V. Cicero, Milano, Bompiani, 2000, p. 67.

[23] Ibid., p. 69.

[24] Ibid. 
qui impiegata per delineare il processo per mezzo del quale lo spirito esce da sé, si realizza e prende coscienza del finito, per poi tornare a sé arricchito di un momento che viene sì negato ma per essere conservato.

La convinzione che l'amore fosse strutturalmente legato alla dialettica che scandisce la vita divina è però una convinzione che Hegel matura ben prima di redigere la Fenomenologia. Già tra il 1797 ed il 1800, nel corso degli anni trascorsi a Francoforte, le suggestioni tratte non solo dal pensiero di Spinoza, recepito con l'essenziale mediazione di Jacobi e Schelling, ma anche dall'assidua lettura dei dialoghi platonici (in special modo il Fedro), spingono Hegel a vedere nell'amore una forza capace di unire in sé una realtà ed il suo opposto, un infinito che assume il finito senza annullarlo in sé, ma conservandolo ad un livello superiore: «Nell'amore» scrive Hegel in un frammento, «rimane ancora il separato, ma non più come separato bensì come unito; ed il vivente sente il vivente». ${ }^{25}$ L'amore, la cui struttura dialettica anticipa per molti versi il movimento di negazione e riconciliazione del soggetto con se stesso che nella Fenomenologia viene attribuito allo spirito, sarà per questo motivo oggetto dell'attenzione costante da parte di Hegel anche durante gli anni della maturità. Nell'Estetica, ad esempio, Hegel osserva che nell'amore, «dal lato del contenuto», ossia senza tener conto della forma che a questo livello non coincide con la compiutezza del concetto

sono presenti i momenti che noi abbiamo indicato come concetto fondamentale dello spirito assoluto: il riconciliato ritorno a se stesso del suo altro [...]. La vera essenza dell'amore consiste nel rinunciare alla coscienza di sé, nell'obliarsi in un altro da sé, ma tuttavia nell'avere e possedere se stesso soltanto in questo perire e dimenticarsi. ${ }^{26}$

Nella struttura dell'amore, che nega ma non annulla il negativo ed il particolare, è pertanto espresso l'assoluto come «mediazione dello spirito con sé»; l'amore rappresenta «il contenuto della soggettività che si media con sé nell'altro» ed è perciò simbolo dell'«assoluto stesso: lo spirito che solo nell'altro spirito è sapere e volere di sé come l'assoluto e possiede il soddisfacimento di

[25] G.W.F. Hegel, Scritti teologici giovanili, tr. E. Mirri, Napoli, Guida, 1989, pp. 558-559. A proposito di questo gruppo di scritti in cui l'amore gioca un ruolo teorico decisivo, Chiereghin osserva: «il vertice della conoscenza è definito da Spinoza come amor Dei intellectualis; Hegel definisce la ragiione come $\pi \lambda \eta \dot{~} \rho \omega \mu$ dell'amore, riflessione e amore congiunti insieme del pensiero. La corrispondenza fra le due espressioni va oltre la coincidenza testuale e rivela l'identità del compito cui è destinato quest'unico strumento capace di definire e di attuare la partecipazione dell'individuo alla vita della totalità divina» (ChIEREghin, L'influenza dello spinozismo, p. 81).

[26] G.W.F. Hegel, Estetica, 2 voll., Torino, Einaudi, 1997, vol. 1, p. 606. 
questo sapere». ${ }^{27}$ Nell'amore divino, in particolare, risassunto nell'unità di finito ed infinito realizzata nella persona di Cristo, diviene evidente «l'idea dell'amore nella sua universalità, l'assoluto, lo spirito della verità nell'elemento e nella forma del sentimento». Libero dalle imperfezioni dell'amore umano, la compiutezza dell'amore divino incarna «la bellezza spirituale, l'ideale, l'identificazione dell'uomo con Dio, con lo spirito, con la verità». Nell'amore divino Hegel vede prefigurato, in termini che richiamano le pagine della parte quinta dell'Etica, tanto la conciliazione tra l'individuo e lo spirito universale quanto, nella misura in cui tale spirito proprio tramite il particolare realizza se stesso, il movimento con cui l'Assoluto si estrania dalla sua iniziale identità per poi recuperare, ad un livello superiore, arricchito da quello che nella Fenomenologia era definito «la serietà, il dolore, la pazienza e il travaglio del negativo», ${ }^{28}$ l'unità con se stesso, in quell'automovimento dialettico che ne scandisce la vita.

Sono accenti che ritornano nella Scienza della logica, dove Hegel ingaggia un serrato confronto con l'ontologia spinoziana, ${ }^{29}$ collocandone il fulcro nel concetto di sostanza, una sostanza che "non giunge neppure alla determinazione dell'esser per sé, e meno che mai poi a quella del soggetto e dello spirito». ${ }^{30}$ Hegel evoca la dottrina dell'amor dei intellectualis, facendola agire contro lo stesso Spinoza, proprio per descrivere il riflettersi in sé dello spirito. In particolare, esponendo la struttura del concetto - che, tramite una «infinita riflessione in se stesso» si rivela «il compimento della sostanza», che assurge a 'soggetto' nella misura in cui si è estraneata ed è poi tornata a se stessa -, ${ }^{31}$ Hegel sottolinea come quelle determinazioni, quelli che per Spinoza erano 'modi' visti come mero non-essere rispetto alla sostanza, assurgono a momenti della vita dell'assoluto:

[...] quello che per la sostanza come tale era un accidentale, la propria mediazione del concetto con se stesso, è la sua propria riflessione immanente. Questa mediazione, che eleva anzitutto l'accidentale alla necessità, è però la relazione manifestata. Il concetto non è l'abisso della sostanza informe ovvero la necessità come identità interna di cose o stati diversi da loro e limitantisi, ma è come assoluta negatività quello che forma, e siccome la determinazione non è come termine, ma è assolutamente come tolta ossia come esser posto, così la parvenza è l'apparire come apparire dell'identico. L'universale è quindi la potenza libera.

[27] Ibid.

[28] Hegel, Fenomenologia dello spirito, p. 69.

[29] Assai utile, a questo proposito, F. Michelini, Sostanza e Assoluto, Bologna, EDB, 2003.

[30] Hegel, Scienza della logica, vol. 1, p. 166.

[31] Ibid., vol. 2, pp. 654-655. 
È se stesso e invade il suo altro; non però come un che di violento, ma come tale che in quello è quieto e presso se stesso. ${ }^{32}$

La «potenza libera» dell'universale, che raggiunge la propria quiete ed è presso se stesso solo assumendo in sé un'alterità che diviene sua parte integrante, viene descritta evocando lo scolio che chiude l'Etica, dove Spinoza si sofferma sulle caratteristiche del saggio, il quale è «difficilmente turbato nell'animo; ma, consapevole di sé, di Dio e delle cose per una certa eterna necessità, non cessa mai di esistere, possedendo sempre il vero acquietamento dell'animo»; ${ }^{33}$ è una condizione, quella del saggio, che fa seguito all'«azione con la quale la mente considera sé stessa come causa», la quale, come affermato poco prima, coincide con «l'azione con la quale Dio, in quanto può esplicarsi mediante la mente umana, contempla sé stesso in associazione all'idea di sé. Perciò [...] questo amore della mente è parte dell'amore infinito con il quale Dio ama sé stesso». In tale condizione l'uomo trova la massima libertà e beatitudine possibili: «Dio, in quanto ama sé stesso, ama gli uomini e, di conseguenza, l'amore di Dio verso gli uomini e l'amore intellettuale della mente verso Dio sono una sola e medesima cosa». ${ }^{34}$ E una terminologia espressamente richiamata da Hegel nel prosieguo del suo ragionamento, dove la «libera potenza» di un Assoluto finalmente riconosciuto come soggetto viene descritta nei termini di «libero amore» ed «illimitata beatitudine, essendo un rapporto di sé al differente solo come a se stesso; nel differente esso è tornato a se stesso». ${ }^{35} \mathrm{Nel}$ descrivere questo libero amore con cui il concetto si realizza ed al contempo si relaziona a se stesso, Hegel fa leva su entrambi i versanti dell'amor dei intellectualis spinoziano. Da un lato, infatti, in esso il particolare si riconosce quale parte dell'universale, il finito si risolve nell'infinito senza però annularsi in esso; diversamente, esso si riconosce quale momento necessario nello sviluppo dell'assoluto:

In quanto la vita, l'Io, lo spirito finito sono certamente anche dei semplici concetti determinati, la loro assoluta risoluzione è in quell'universale, che si ha da intendere come concetto veramente assoluto, come idea dello spirito infinito, il cui esser

[32] Ibid., vol. 2, pp. 682-683.

[33] B. SpInoza, Etica, parte V, prop. XLII, scol., p. 1086.

[34] Ibid., parte V, prop. XXXVI, p. 1079.

[35] Hegel, Scienza della logica, vol. 2, p. 683; commenta a questo proposito Angelica Nuzzo: «Die Ewigkeit der Idee ist damit zweimal ausgesprochen, doch ist am wichtigsten, dass sie in der Form eines ewigen Selbstbezugs, in dem sich die Idee als absoluter Geist "betaetigt, erzeugt und geniesst", zum Ausdruck kommt. Diese abschliessende Gestalt entspricht Spinozas amor Dei intellectualis. Die erzeugende Kraft des amor dei intellectualis ersetzt nun die Produktivitat der selbstbezueglichen caus sui. Die Liebe, mit der Gott sich selbst und zugleich den Menschen liebt, findet im selbstbewussten Denken - der sich wissenden Vernunft - statt» (A. Nuzzo, Spinozas Amor dei intellectualis, p. 259). 
posto è la realtà infinita, trasparente, nella quale esso concetto contempla la sua creazione e in questa sua creazione se stesso. ${ }^{36}$

In questo senso, il finito non è più, al pari del modus spinoziano, ${ }^{37}$ una specificazione esteriore rispetto alla vita dell'universale, un non-essere che cade fuori dall'unità della sostanza; anzi, ed è questo l'altro versante della posizione hegeliana, è l'Assoluto stesso che, nel finito, assume concretezza e si realizza come "potenza creativa [...] negatività assoluta che si riferisce a se stessa». ${ }^{38}$ La vita dello spirito è assume così le sembianze di un'amor dei intellectualis la cui fisionomia si chiarisce a partire dal duplice valore del genitivo dei, che è al contempo soggettivo e oggettivo: oggettivo nella misura in cui descrive l'amore dell'uomo verso Dio e, da qui, l'inverarsi del finito in un infinito che non lo sopprime, ma lo conserva dentro di sé e per suo tramite anzi si concretizza; soggettivo, perché l'amore che Dio prova verso di sé diviene metafora del relazionarsi del concetto a se stesso. In conclusione, Hegel attinge alla parte quinta dell'Etica per esprimere, in pagine quantomai efficaci, quel movimento continuo di estraniazione e riconciliazione, soppressione e conservazione, che è poi la caratteristica definitoria di un Assoluto la cui teorizzazione rappresenta, d'altra parte, un cosciente tentativo di porsi oltre la stessa ontologia spinoziana. Nel quadro di quello che è, per dirla in una battuta, un complesso sforzo volto a correggere e superare Spinoza per mezzo di strumenti attinti allo stesso Spinoza, Hegel sfrutta il potenziale metaforico e speculativo di un' intuizione che, in ambito storico-filosofico, si era rivelata vincolata ai limiti della filosofia in cui era maturata. Innestandola nel proprio sistema ed adattandola ai propri specifici scopi argomentativi, Hegel però ripensa, su basi del tutto nuove, la dottrina dell'amor dei intellectualis, che diviene così la cartina di tornasole del passaggio dalla sostanza al soggetto, dall'essere al concetto, dal «vero» all'«intero vero».

Salvatore Carannante is is post-doc fellow at the Istituto Nazionale di Studi sul Rinascimento (Florence, Italy)

RESEARCH INTERESTS:

History of Platonic tradition; Renaissance philosophy; German idealism

[36] Hegel, Scienza della logica, vol. 2, p. 684.

[37] «In quell'assoluto che è soltanto l'identità immobile, l'attributo, come il modo, è solo come dileguantesi, non come tale che diviene, per modo che così anche quel dileguarsi prende il suo cominciamento positivo soltanto dal di fuori» (Ibid., p. 605).

[38] Ibid., p. 685. 
AdDress:

Salvatore Carannante

c/o Scuola Normale Superiore

Piazza dei cavalieri 7

56126 Pisa (Italy)

ReCEnt Publications:

Giordano Bruno e la caccia divina, Pisa: Edizioni della Normale 2014.

«Quell'intelletto artefice...». Causa, principio ed intelletto universale nel De la causa di Bruno, «Rinascimento», LII (2012), pp. 59-96. 